\title{
Sustainability of a Primary Care-Driven eConsult Service
}

\author{
Clare Liddy, MD, MSc, CCFP 1,2 \\ Isabella Moroz, $\mathrm{PbD}^{1}$ \\ Amir Afkham, BEng (Hons) ${ }^{3}$ \\ Erin Keely, MD, FRCPC ${ }^{4,5}$ \\ ${ }^{1} \mathrm{CT}$ Lamont Primary Health Care Research \\ Centre, Bruyère Research Institute, Ottawa, \\ Ontario, Canada \\ ${ }^{2}$ Department of Family Medicine, Univer- \\ sity of Ottawa, Ottawa, Ontario, Canada \\ ${ }^{3}$ The Champlain Local Health Integration \\ Network, Ottawa, Ontario, Canada \\ ${ }^{4}$ Department of Medicine, University of \\ Ottawa, Ottawa, Ontario, Canada \\ ${ }^{5}$ Division of Endocrinology/Metabolism, \\ The Ottawa Hospital, Ottawa, Ontario, \\ Canada
}

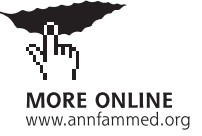

Conflicts of interest: authors report none.

\section{CORRESPONDING AUTHOR}

Clare Liddy, MD, MSC

Bruyère Research Institute

43 Bruyère St, Annex E, Room 106

Ottawa, ON K1N 5C8

cliddy@bruyere.org

\begin{abstract}
PURPOSE Excessive wait times for specialist appointments pose a serious barrier to patient care. To improve access to specialist care and reduce wait times, we launched the Champlain BASE (Building Access to Specialists through eConsultation) eConsult service in April 2011. The objective of this study is to report on the impact of our multiple specialty eConsult service during the first 5 years of use after implementation, with a focus on growth and sustainability.
\end{abstract}

METHODS We conducted a cross-sectional study of all eConsult cases submitted between April 1, 2011 and April 30, 2016, and measured impact with system utilization data and mandatory close-out surveys completed at the end of each eConsult. Impact indicators included time interval to obtain specialist advice, effect of specialist advice on the primary care clinician's course of action, and rate of avoidance of face-to-face visits.

RESULTS A total of 14,105 eConsult cases were directed to 56 different medical specialty groups, completed with a median response time of 21 hours, and $65 \%$ of all eConsults were resolved without a specialist visit. We observed rapid growth in the use of eConsult during the study period: 5 years after implementation the system was in use by 1,020 primary care clinicians, with more than 700 consultations taking place per month.

CONCLUSIONS This study presents the first in-depth look at the growth and sustainability of the multispecialty eConsult service. The results show the positive impact of an eConsult service and can inform other regions interested in implementing similar systems.

Ann Fam Med 2018;16:120-126. https://doi.org/10.1370/afm.2177.

\section{INTRODUCTION}

$\mathrm{E}$ xcessive wait times for medical specialist care is a serious issue in Canada. ${ }^{1,2}$ In a recent Commonwealth Fund survey, Canada placed last in patient access to care among 11 participating countries. ${ }^{1}$ Prolonged waiting for care is more than a simple frustration for patients. It can result in serious and long-term consequences by affecting a patient's ability to carry out daily activities and by causing increased worry, pain, and deterioration in overall health. ${ }^{2}$ Furthermore, delayed access to care by specialists can result in delayed diagnosis, duplication of services, and dissatisfaction among primary care clinicians. ${ }^{3}$

There is opportunity to improve access to specialist care with the use of innovative electronic health platforms such as electronic consultation (eConsult). The eConsult service links primary care clinicians and specialists, enables the specialist to give advice directly to the primary care clinician, and often negates the need for a face-to-face visit. ${ }^{4-6}$ Research suggests that electronic services can increase access to specialist care, reduce wait times, and improve communication between primary care clinicians and specialists. ${ }^{7-10}$

In 2010, we created the Champlain BASE (Building Access to Specialists through eConsultation) eConsult service to address the problem of excessive wait times for nonurgent specialist appointments. ${ }^{4}$ We received a grant to develop a proof-of-concept service that ran from January 2010 to April 2011. Our partner, the Champlain Local Health Integration 
Network, provided use of their SharePoint platform (hosted at the Winchester District Memorial Hospital) at no additional cost. ${ }^{4}$ Privacy impact and threat risk assessments were conducted to ensure eConsult complied with Ontario's Personal Health Information Protection Act. The Canadian Medical Protective Association (CMPA) was contacted to identify any issues pertaining to liability. The assessments revealed that the eConsult service met all privacy standards and the CMPA confirmed that it fell within the parameters of liability already established for informal (hallway) consultations in which primary care clinicians seek advice on a patient's care from specialist colleagues. ${ }^{11}$

After the successful proof-of-concept phase, we obtained funding from local partners and the Ministry of Health of Ontario/eHealth Ontario to launch a full pilot study in 2011. ${ }^{12}$ The eConsult service was offered to a wider range of primary care clinicians (mostly in our health region, but also in other geographic areas). The eConsult service now offers access to specialty advice for 2.4 million people in Ontario and is poised for further expansion in Ontario and across Canada. ${ }^{13,14}$

Canada has been referred to as a country of perpetual pilot projects, where innovative health care practices are often implemented but fail to expand beyond their initial jurisdiction or sustain activity beyond their initial funding period. ${ }^{15}$ Dissemination of results and ongoing evaluation of new services are important to (1) better understand implementation processes, and (2) ensure that early results are maintained beyond the pilot stage.

The goal of this paper is to report on the growth and outcomes of the eConsult service, the first Canadian service of its kind to extend beyond its pilot phase. This detailed analysis may serve as a blueprint for future implementations, and will be of use to stakeholders interested in rolling out similar services in other jurisdictions.

\section{METHODS}

\section{Study Design}

This is a cross-sectional study of all cases submitted by primary care clinicians registered to use the eConsult service between April 1, 2011 and April 30, 2016. This study was approved by the Ottawa Health Science Network Research Ethics Board (Protocol \# 2009848-01H)

\section{Setting}

The geographic setting is predominantly a large health region in Eastern Ontario, Canada with a population of 1.3 million people who have referral patterns comparable to the rest of Canada. ${ }^{16}$ The region has 1 urban referral center serving a geographic area of $16,000 \mathrm{~km}^{2}$ with outlying rural communities up to 2 hours away by car.

\section{The Champlain BASE eConsult Service}

The eConsult service is provided through a secure web-based application that enables asynchronous consultation between primary care clinicians and specialists. Primary care clinicians log into the application, enter a patient-specific clinical question into an electronic form, and select the most appropriate specialty group from a drop-down list. Primary care clinicians can attach files containing supplementary information such as digital images, test results, and health histories. A designated assigner directs the question to a specialist from the chosen specialty group based on availability and caseload. The specialist is notified by e-mail and is expected to respond within 1 week. The service is easy to use and requires only a standard computer with Internet access. User registration and orientation typically takes 30 minutes. Upon submission of the response, the specialist is asked to report the amount of time spent and paid $\$ 200$ per hour, prorated. Primary care clinicians were not originally compensated, but recently adopted new fee codes allow primary care clinicians to bill $\$ 16$ per eConsult. ${ }^{17}$ The proof-of-concept project was funded entirely by research grants, but the pilot study is supported by a combination of research grants and regional and provincial funding.

A key feature of our model is that it is set up as a managed service, which means expectations for quality and timeliness of responses are established up front and monitored on an ongoing basis. The goal is not to make every specialist accessible through this service, but rather to ensure that a wide range of specialties are available so that primary care clinicians can meet the needs of their patients, that each specialty is served by an appropriate number of specialists to handle the volume of cases as utilization grows, that each specialist is committed to providing a high-quality response within 1 week, and that the specialist recognizes and enjoys the educational aspect of the service. Additionally, options can be set up to support focus on some specialties in specific regions, to divide specialties into French/English versions, and to provide subcategorization within a specialty. User's technical issues are to be resolved the same day and requests for service enhancement are to be acknowledged within 2 business days and implemented as appropriate.

Our managed service approach is distinct from other eConsult services, which are usually set up as direct links between individual primary care clinicians and specialists. The direct access type of services enable primary care clinicians to search a directory, 
find a specialist's contact information, and reach out to them via a secure channel. The primary care clinician may have many options to choose from and may not know if the specialist is available to respond in a timely manner, has the breadth and depth of experience needed, or has a history of providing high-quality responses in electronic consultation systems. In contrast, our managed service approach simplifies the process for primary care clinicians by allowing them to choose a specialty group rather than browsing a list of individual specialists. Once a primary care clinician submits a case, it is routed to an available specialist who has the necessary qualifications. The system monitors the timeliness of the responses and the primary care clinician's satisfaction for each case. Feedback is provided to specialists as appropriate.

\section{Participants}

Participants included the primary care clinicians and medical specialists who registered to use the eConsult service, which is available to all physicians and nurse practitioners in Ontario. Utilization data is from users who completed at least 1 eConsult during the study period. Needs of the primary care clinicians served as the primary criteria for specialist recruitment, with specialists from new specialty groups added in response to user demand on an ongoing basis. In this paper we report on cases submitted to medical specialties, not to allied health professionals which have been reported separately.

\section{Data and Variables}

Utilization data are collected in real time and include the number of eConsults a primary care clinician has submitted, the specialty groups referred to, the specialist's response time, and the case outcome. Case outcomes can include: (1) a recommendation for the patient's care, (2) a request for additional patient information, or (3) a recommendation that the patient receive a formal referral. The service also logs the complete exchange between primary care clinician and specialist.

The outcomes for impact analysis are collected with a brief 5 -field close-out survey completed by primary care clinicians at the conclusion of each case (Supplemental Appendix 1, available at http://www. annfammed.org/content/16/2/120/suppl/DC1/). The survey asks primary care clinicians to assess: (1) the outcome of the eConsult for the patient, (2) whether or not a referral was contemplated before/after the eConsult, (3) the value of the eConsult for the patient and (4) the value of the eConsult to the primary care clinician, and (5) any additional comments about the service. For this report, we focused on primary care clinicians' responses to questions 1 and 2 .
We used descriptive statistics to characterize the primary care clinicians, specialists, and patients. Response times do not follow a normal distribution, therefore medians and seventy-fifth percentiles were calculated.

\section{RESULTS}

A total of 1,020 primary care clinicians registered for the eConsult service and 726 became users by completing at least 1 eConsult. Primary care clinicians submitted 14,105 eConsult cases to 56 of the 63 specialties available during the study. Primary care clinician, specialist, and patient demographics and service utilization information are shown in Table 1.

The specialty groups receiving the highest number of eConsults were dermatology (17.11\%), obstetrics/ gynecology $(7.02 \%)$, hematology $(6.99 \%)$, endocrinology (6.93\%), cardiology (6.27\%), and neurology $(5.98 \%)$. Specialists responded in a median of 21 hours, with $75 \%$ of cases answered within 3 days. The selfreported billing time for specialists was less than 10 minutes in $48 \%$ of cases, $10-15$ minutes in $32 \%, 15-20$ minutes in $15 \%$, and over 20 minutes in $4 \%$.

\section{Table 1. Primary Care Clinician, Specialist, Patient, and Utilization Characteristics of the Champlain BASE eConsult Service}

\begin{tabular}{|c|c|}
\hline Characteristics & Distribution \\
\hline \multicolumn{2}{|l|}{ Primary care clinicians $(n=1,020)$} \\
\hline \multicolumn{2}{|l|}{ Type, No. (\%) } \\
\hline Nurse practitioner & $160(16)$ \\
\hline Family physician & $860(84)$ \\
\hline Sex, female, No. (\%) & $701(69)$ \\
\hline Practice location, rural & $107(69)$ \\
\hline \multicolumn{2}{|l|}{ Specialists $(n=106)$} \\
\hline Sex, female, No. (\%) & $47(44)$ \\
\hline \multicolumn{2}{|l|}{ Patients $(n=14,105)$} \\
\hline \multicolumn{2}{|l|}{ Age group, No. (\%) } \\
\hline$<18 y$ & $2,249(16)$ \\
\hline $18-64$ y & $8,824(63)$ \\
\hline$\geq 65$ y & $3,032(21)$ \\
\hline Sex, female, No. (\%) & $8,750(62)$ \\
\hline \multicolumn{2}{|l|}{ Response time } \\
\hline $\begin{array}{l}\text { eConsult creation to specialist response, } \\
\text { median ( } 75^{\text {th }} \text { percentile), } \mathrm{h}\end{array}$ & $20.75(80.98)$ \\
\hline $\begin{array}{l}\text { eConsult creation to primary care clinician } \\
\text { close out, median ( } 75^{\text {th }} \text { percentile), d }\end{array}$ & $5.09(10.98)$ \\
\hline \multicolumn{2}{|l|}{$\begin{array}{l}\text { Self-reported specialist response time, } \\
\text { No. }(\%)(n=14,105)\end{array}$} \\
\hline$<10 \min$ & $6,796(48)$ \\
\hline $10-15 \min$ & $4,566(32)$ \\
\hline $15-20 \mathrm{~min}$ & $2,127(15)$ \\
\hline$>20 \min$ & $616(4)$ \\
\hline
\end{tabular}


Figure 1a. Average number of eConsults completed per primary care clinician for users of the Champlain BASE eConsult service as function of their registration year.

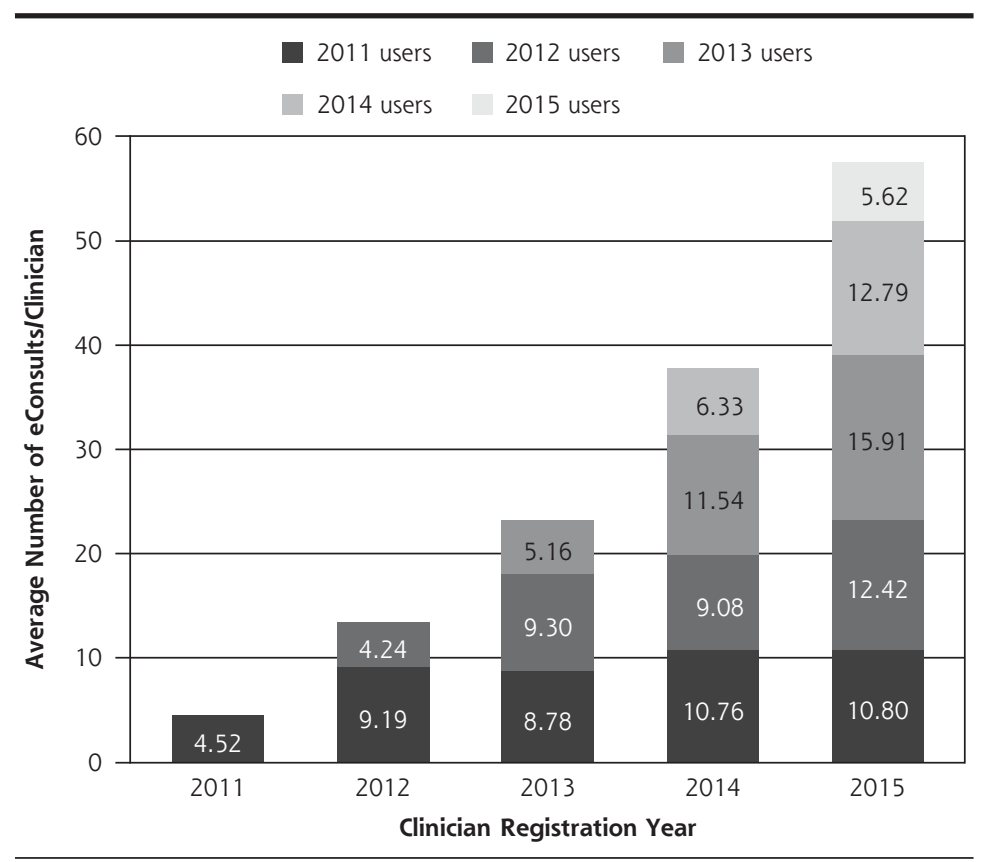

BASE $=$ Building Access to Specialists through eConsultation.

Note: User denotes an individual who used the service at least once in the year indicated.

Figure $1 \mathrm{~b}$. Number of primary care clinician users of the Champlain BASE eConsult service in relation to all primary care clinicians who registered for the service each year.

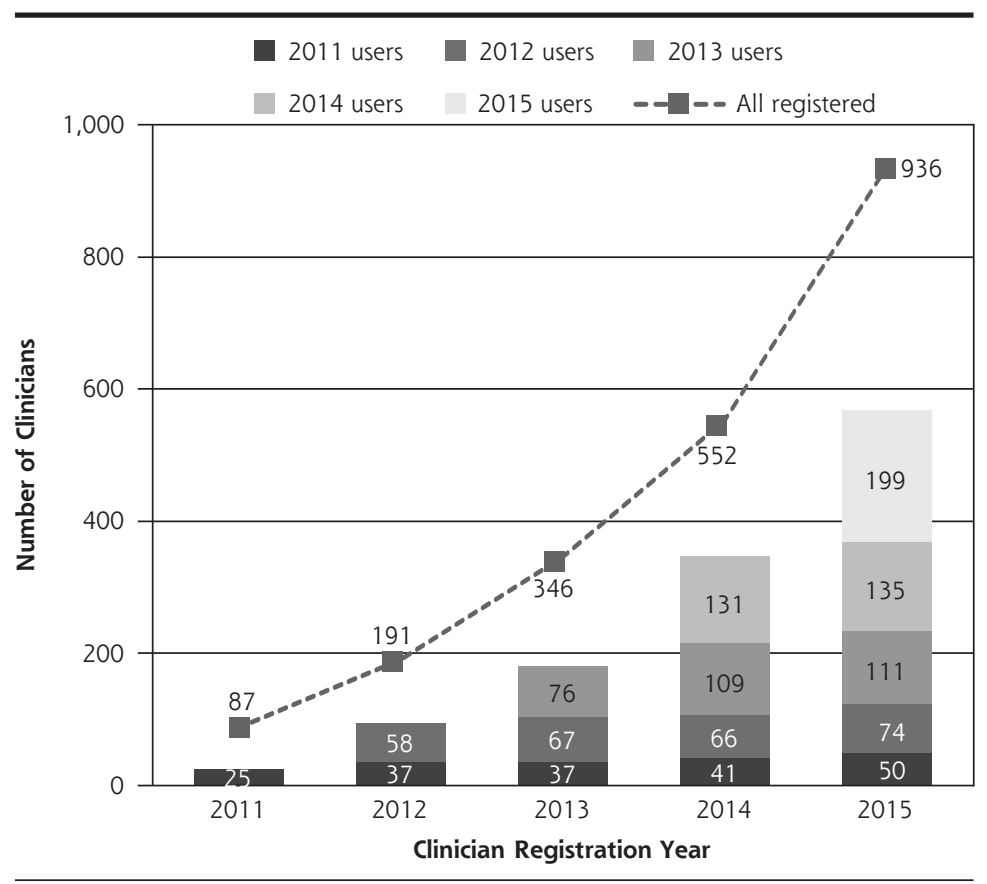

BASE $=$ Building Access to Specialists through eConsultation.

Note: User denotes an individual who used the service at least once in the year indicated.

\section{Service Growth}

The service experienced exponential growth during the 5 -year pilot study, increasing from 4 cases closed in April 2011 to 769 cases closed in April 2016. A breakdown of the average number of eConsults submitted annually by active users who registered for the service in each of the 5 years of the study is presented in Figure 1a. Primary care clinicians submitted a similar number of cases in their first year regardless of the year they joined the service, with average annual number of cases ranging from 4.24 to 6.33 per primary care clinician. In the second year case numbers approximately doubled and then increased more gradually in subsequent years. Figure $1 \mathrm{~b}$ shows the number of users of eConsult in each year following registration in relation to all registrants.

The eConsult service experienced a substantial expansion in specialist engagement over the study period: from 36 specialists representing 16 specialty groups in 2011 to 132 specialists representing 63 specialty groups by April 30, 2016. Twelve specialists withdrew from the service during this time period citing heavy workload $(\mathrm{n}=8)$, moving $(\mathrm{n}=3)$, and job change $(\mathrm{n}=1)$ as their reasons.

\section{Impact on Primary Care Clinician Behavior}

Across specialty groups, primary care clinicians reported receiving advice for a new or additional course of action in $57 \%$ cases and confirmatory advice in $40 \%$ of cases. A face-to-face specialist visit was originally planned but avoided as a result of eConsult in $39.56 \%$ of cases, originally planned and still needed in $24.64 \%$, neither originally contemplated nor ultimately needed in $27.98 \%$, and not originally contemplated but instigated based on the specialist's advice in $3.53 \%$. The remaining cases were reported as having no benefit $(1.82 \%)$ or other results $(2.47 \%)$. The eConsult service's impact on primary care clinician course of action and need for a face-toface consultation for individual specialties is shown in Figures 2a and 2b. 


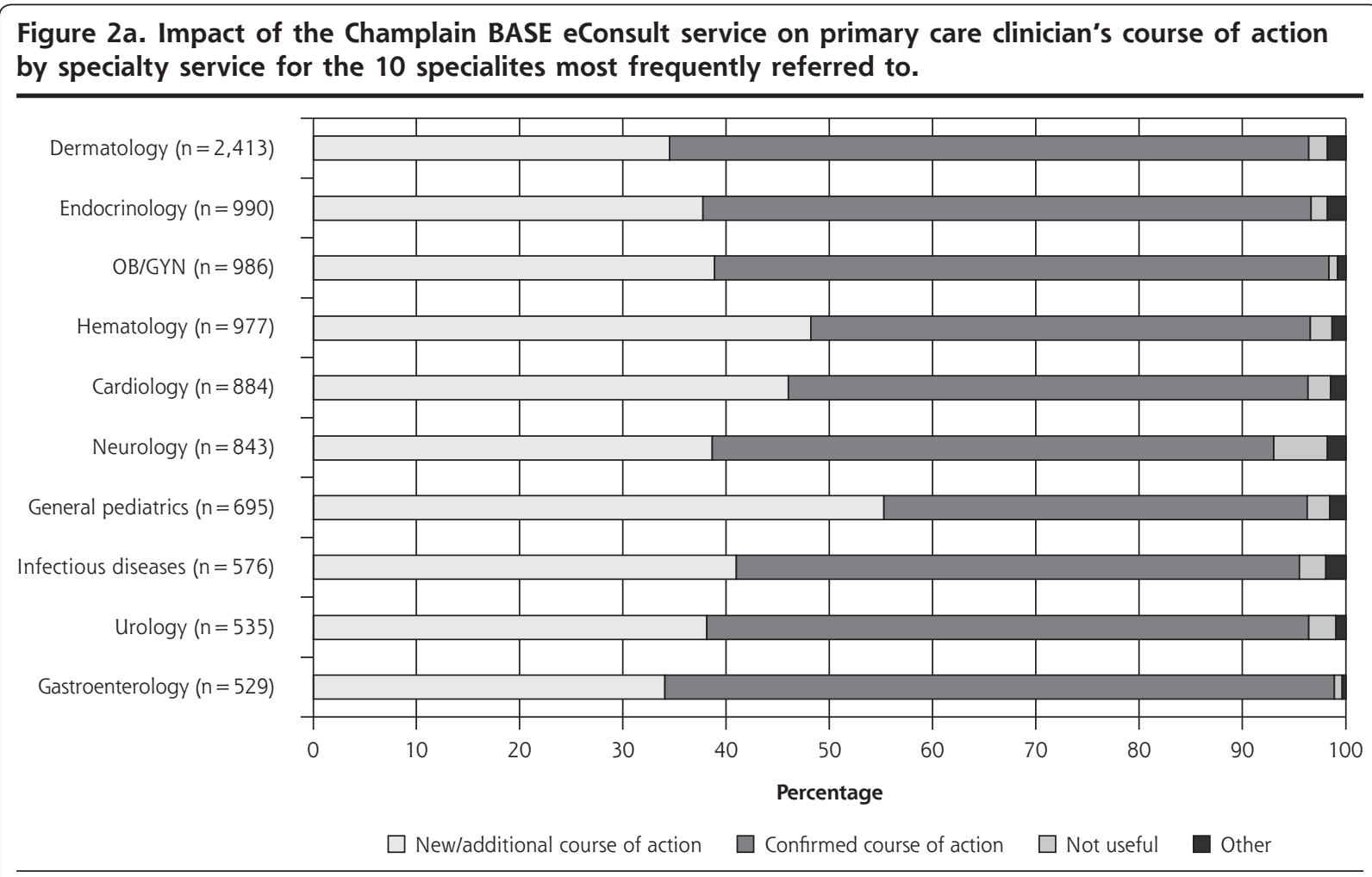

$\mathrm{BASE}=$ Building Access to Specialists through eConsultation; OB/GYN = obstetrics and gynecology

\section{DISCUSSION}

The success of the eConsult service is a rare example of a local innovation that managed to expand beyond its pilot phase and grow at the regional, provincial, and national level. Many of the metrics we used to measure eConsult (for example, the rate of referral avoidance, specialist response time, and primary care clinician-reported value) were remarkably stable over time. By the end of the study, roughly $80 \%$ of primary care clinicians in our health region had adopted the service, establishing its popularity in the medical community. Much of the previous literature on eConsult is limited to 1 specialty service (commonly dermatology). Our results provide a unique look at eConsult's general impact on patient care across multiple specialty groups, including those with complex or atypical cases (eg, psychiatry, chronic pain). ${ }^{8,9}$

The rapid growth in numbers of primary care clinicians registering for and using the eConsult service can be partially explained by Rogers' diffusion of innovation theory ${ }_{1}^{18}$ which identifies several attributes as critical to facilitating adoption of innovations: relative advantage, compatibility, complexity, observability, and trialability. The relative advantages of the eConsult compared to traditional referral methods are the rapid access to specialist's expertise (in days instead of months), improvement in patient care, and avoidance of expensive and sometimes unnecessary face-to-face visits. The managed service approach ensures participants' expectations are established at the beginning and consistently met or exceeded. Also, eConsults simplify and improve the referral process by providing an effective methiod of communication between primary care clinicians and specialists. Consequently, most primary care clinicians receive a response from specialists in less than a day, and the specialist is most often able to complete the response in less than 10 minutes. ${ }^{12}$

The benefits of the eConsult service are aligned with the needs of policymakers, primary care clinicians, specialists, and patients, which helps to create to a receptive context for and facilitate adoption of innovations. ${ }^{19}$ The access barriers that eConsult sought to address (long wait times and geographic distance) are not unique to our health region, and have been identified as strategic priorities in many provinces and territories. Consequently, there is great interest in eConsult services across the country. The eConsult service recently joined a national eCollaborative led by the Canadian Foundation for Healthcare Improvement in partnership with the Royal College of Physicians and Surgeons, Canada College of Family Physicians, and Canada Health Infoway, that supports the scale up and implementation of the eConsult service and other 


\section{Figure 2b. Impact of the Champlain BASE eConsult service on the need for a face-to-face referral by specialty service for the 10 specialties most frequently referred to.}

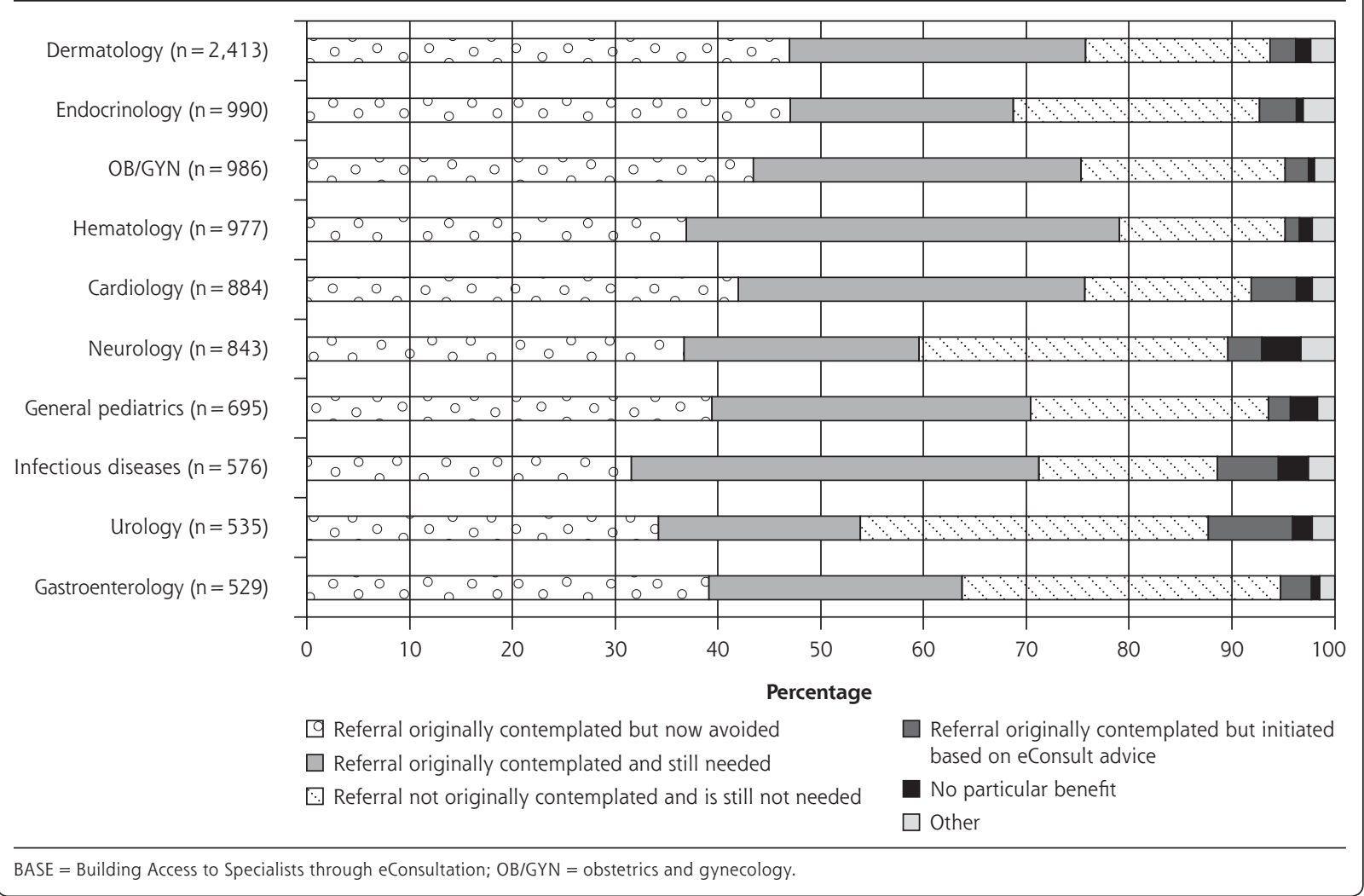

access initiatives in 10 jurisdictions across Canada. ${ }^{14}$ Unlike other eHealth solutions which failed to realize their potential in real world settings, ${ }^{9,20,21}$ our eConsult service has been developed with special consideration for local contexts and in partnership with provincial and national organizations. These considerations and partnerships have proven critical to the successful growth, scale up, and national expansion of the eConsult service. Recent data from the Los Angeles Safety-Net Program demonstrated a rapid and successful adoption of an eConsult system between 2012 and 2015 that improved access to specialists for a large disadvantaged population with historically poor access to specialty care. ${ }^{10}$ These results are comparable to ours in terms of rapid growth and sustainability of the service and illustrate the potential of technology-enabled communication between primary care clinicians and specialists for underserved populations.

Our study has several strengths, one of which is that the eConsult service has one of the largest menus of specialties found in this type of service worldwide. The close-out survey has yielded data that allows us to compare referral patterns, monitor key performance indicators related to quality and timeliness, and address issues promptly. Additionally, because data are col- lected immediately on closure of the case, there is less chance of recall bias. Finally, the data provide insight to the impact on physician behavior and avoidance of face-to-face visits with the specialists from the perspective of the primary care clinician.

Our study has some limitations. It is descriptive in nature and was not equipped to examine the impact on wait times. The participating primary care clinicians were self-selected and we did not have access to the total number of referrals in our health region, which limits our ability to understand impact of eConsult on a population level although this is work in progress.

The eConsult service is an innovative and effective way of improving access to specialty care for Canadians. We have documented rapid uptake, adoption, and consistently stable impact in terms of avoidance of specialist visits. This illustrates the potential of the eConsult service to affect wait times for specialty care once integrated into the existing workflows for specialty referrals within the clinics. These results provide a better understanding of referral patterns within an eConsult service and can be used to inform other regions interested in implementing similar systems.

To read or post commentaries in response to this article, see it online at http://www.AnnFamMed.org/content/16/2/120. 
Key words: primary care; electronic consultation; eConsult; referral; access to care; wait times; telemedicine; health equity

Submitted July 14, 2017; submitted, revised, October 6, 2017; accepted October 18, 2017.

Funding support: Funding for this project was provided by the Ontario Ministry of Health and Long-Term Care, INSPIRE-PHC Applied Health Research Question. The authors affirm their independence from these funders. The funders played no part in the study design, collection, analysis, or interpretation of the data, in the writing of the report, or in the decision to submit the article for publication.

Previous presentations: This study was presented in part at the Trillium Primary Health Care Research Day; May 31, 2017; Toronto, Ontario; and presented in part at the NAPCRG Annual Meeting; November 17-21, 2017; Montreal, Quebec.

Acknowledgments: We would like to thank the primary care clinicians and specialists who participated in the service. In addition, we extend our gratitude to the Winchester District Memorial Hospital who hosts the servers, and Justin Joschko for his assistance in editing the manuscript and preparing it for publication.

Supplementary materials: Available at http://www.AnnFamMed. org/content/16/2/120/suppl/DC1/.

\section{References}

1. Canadian Institute for Health Information. How Canada Compares: Results From The Commonwealth Fund's 2016 International Health Policy Survey of Adults in 11 Countries. Ottawa, ON: Canadian Institute for Health Information; 2012. https://www.cihi.ca/sites/default/ files/document/text-alternative-version-2016-cmwf-en-web.pdf. Published 2012. Accessed Jul 14, 2017.

2. Canadian Intitute for Health Information. Health Care in Canada, 2012: A Focus on Wait Times. Ottawa, ON: Canadian Institute for Health Information; 2012. https://secure.cihi.ca/free_products/ HCIC2012-FullReport-ENweb.pdf. Published Nov 29, 2912. Accessed Jul 14, 2017.

3. Barua B, Esmail N. Waiting Your Turn: Wait Times for Health Care in Canada. Vancouver, CA: Fraser Institute; 2013. https://www. fraserinstitute.org/sites/default/files/waiting-your-turn-2013.pdf. Published Oct 2013. Accessed Jul 14, 2017.

4. Liddy C, Rowan MS, Afkham A, Maranger J, Keely E. Building access to specialist care through e-consultation. Open Med. 2013; 7(1):e1-e8

5. Kim-Hwang JE, Chen AH, Bell DS, Guzman D, Yee HF Jr, Kushel MB. Evaluating electronic referrals for specialty care at a public hospital. J Gen Intern Med. 2010;25(10):1123-1128.

6. Stoves J, Connolly J, Cheung CK, et al. Electronic consultation as an alternative to hospital referral for patients with chronic kidney disease: a novel application for networked electronic health records to improve the accessibility and efficiency of healthcare. Qual Saf Health Care. 2010;19(5):e54.
7. Straus SG, Chen AH, Yee H Jr, Kushel MB, Bell DS. Implementation of an electronic referral system for outpatient specialty care. AMIA Annual Symposium Proceedings 2011;2011:1337-46.

8. Vimalananda VG, Gupte G, Seraj SM, et al. Electronic consultations (e-consults) to improve access to specialty care: a systematic review and narrative synthesis. J Telemed Telecare. 2015;21(6):323-330.

9. Liddy C, Drosinis P, Keely E. Electronic consultation systems: worldwide prevalence and their impact on patient care-a systematic review. Fam Pract. 2016;33(3):274-285.

10. Barnett ML, Yee HF Jr, Mehrotra A, Giboney P. Los Angeles safetynet program eConsult system was rapidly adopted and decreased wait times to see specialists. Health Aff (Millwood). 2017;36(3): 492-499.

11. Canadian Medical Protective Association. CMPA Assessment of eConsult. Ottawa, ON: CMPA; 2015. https://dropbox.otn.ca/econsult-help/ cmpa-assessment-econsult.pdf. Accessed Sep 22, 2017.

12. Keely E, Liddy C, Afkham A. Utilization, benefits, and impact of an e-consultation service across diverse specialties and primary care providers. Telemed J E Health. 2013;19(10):733-738.

13. Newfoundland and Labrador Medical Association. E-Consult. St. John's, NL: NLMA; 2017. http://www.nlma.nl.ca/Physicians/ eConsult/. Accessed Jul 14, 2017.

14. Canadian Foundation for Healthcare Improvement. Leading Canadian Healthcare Organizations Team Up to Tackle Long waits for Specialist Care. Ottawa, ON: CHFl; 2017. http://www.cfhi-fcass.ca/ NewsAndEvents/NewsReleases/Newsltem/2017/03/07/leadingcanadian-healthcare-organizations-team-up-to-tackle-long-waits-forspecialist-care/. Accessed Jul 14, 2017.

15. Bégin M, Eggertson L, Macdonald N. A country of perpetual pilot projects. CMAJ. 2009;180(12):1185, E88-E89.

16. Champlain Local Health Integration Network. Population Characteristics for Champlain Health Link Areas. Ottawa, ON: Champlain LHIN; 2014. http://www.champlainlhin.on.ca/ /media/sites/champlain/ Accountability/Integration/HL\%20Docs/201410ChHLAsPopCharEN. pdf?la =en. Published Oct 2014. Accessed Jul 14, 2017.

17. Medical Systems JCL. E-consultations: K738 and K739. Oakville, ON: JCL Medical Systems; 2016. https://jclmedicalbilling.ca/ohip-billingcodes/e-consultations-k738-and-k739/. Published May 2, 2016. Accessed Sep 22, 2017.

18. Rogers E. Diffusion of Innovations. New York, NY: Free Press; 2003.

19. Greenhalgh T, Robert G, Macfarlane F, Bate P, Kyriakidou O. Diffusion of innovations in service organizations: systematic review and recommendations. Milbank Q. 2004;82(4):581-629.

20. Ammenwerth E, Talmon J, Ash JS, et al. Impact of CPOE on mortality rates-contradictory findings, important messages. Methods Inf Med. 2006;45(6):586-593.

21. Liddy C, Hogel M, Blazkho V, Keely E. The current state of electronic consultation and electronic referral systems in Canada: an environmental scan. Stud Health Technol Inform. 2015;209:75-83. 